\title{
Effective GDNF brain delivery using microspheres-A promising strategy for Parkinson's disease
}

\author{
E. Garbayo ${ }^{1}$, C.N. Montero-Menei ${ }^{2}$, E. Ansorena ${ }^{1}$, J.L. Lanciego ${ }^{3}$, M.S. Aymerich ${ }^{3}$, M.J. \\ Blanco-Prieto ${ }^{1}$
}

${ }^{1}$ Department of Pharmacy and Pharmaceutical Technology, University of Navarra, Spain

2 Inserm U646, Laboratoire d'Ingénierie de la Vectorisation Particulaire; Université d'Angers, France ${ }^{3}$ Department of Neurosciences, Center for Applied Medical Research (CIMA and CIBERNED) University of Navarra Medical College, Spain

* To whom correspondence should be addressed:

Dr. María J. Blanco Prieto

Department of Pharmacy and Pharmaceutical Technology, School of Pharmacy, University of Navarra, C/Irunlarrea 1, E-31080 Pamplona, Spain

Office phone: + 34948425600 ext. 6519

Fax: + 34948425649

e-mail:mjblanco@unav.es

\begin{abstract}
Glial cell line-derived neurotrophic factor (GDNF) has shown promise in the treatment of neurodegenerative disorders of basal ganglia origin such us Parkinson's disease (PD). In this study, we investigated the neurorestorative effect of controlled GDNF delivery using biodegradable microspheres in an animal model with partial dopaminergic lesion. Microspheres were loaded with N-glycosylated recombinant GDNF and prepared using the Total Recirculation One-Machine System (TROMS). GDNF-loaded microparticles were unilaterally injected into the rat striatum by stereotaxic surgery two weeks after a unilateral partial 6-OHDA nigrostriatal lesion. Animals were tested for amphetamineinduced rotational asymmetry at different times and were sacrificed two months after microsphere implantation for immunohistochemical analysis. The putative presence of serum IgG antibodies against rat glycosylated GDNF was analyzed for addressing safety issues. The results demonstrated that GDNF-loaded microspheres, improved the rotational behavior induced by amphetamine of the GDNF-treated animals together with an increase in the density of TH positive fibers at the striatal level. The developed GDNF-loaded microparticles proved to be suitable to release biologically active GDNF over up to 5 weeks in vivo. Furthermore, none of the animals developed antibodies against GDNF demonstrating the safety of glycosylated GDNF use.
\end{abstract}

Key words: GDNF, PLGA microparticles, TROMS, serum IgG antibodies against GDNF, Parkinson's disease.

\section{Introduction}

Neurotrophic factors have emerged as promising tools for the treatment of a wide variety of neurodegenerative diseases. Among them, the glial cell-line derived neurotrophic factor (GDNF) was selected as the most suitable candidate for the treatment of Parkinson's disease (PD) due to its strong trophic effect on the dopaminergic system $[1,2]$. The initial successful results obtained in relevant animal 
models of the disease led to different clinical trials in PD patients. The outcome obtained in two independent Phase I clinical trials known as the "Bristol" and "Kentucky" studies [3-5], using direct intraputaminal infusion of naked nonglycosylated GDNF through mini-pumps, was not further confirmed by a double-blind Phase II study using a similar strategy [6]. Several safety concerns were reported, such as the appearance of blocking antibodies against GDNF, together with the presence of unexpected cerebellar damage in a toxicology study carried out in parallel in primates treated with high doses of GDNF. However, the main reason for the discontinuation of the study was the failure of reaching the primary endpoint [6]. Differences in GDNF doses, catheter size and infusion methods may have resulted in different GDNF spread and bioavailability. Recently, the statistical design of this Phase II study was also questioned [7].

Several strategies have been used for GDNF release in the central nervous system (CNS). A catheter connected to an infusion pump has already been used in PD patients [3-6]. This method has some disadvantages, such as the need of high concentrations of the neurotrophic factor as well as pump refilling, together with limited tissue diffusion of the delivered protein [8]. Another feasible option is the use of gene therapy using different strains of modified viruses carrying the GDNF gene [9-11]. This approach also presents some disadvantages such as the lack of control of the duration of the transgene expression, the viral spread outside the target area, and the difficulties in calculating the exact amount of GDNF produced from the viral-infected neurons. Finally, a different alternative would be the use of cell therapy strategies using cells genetically engineered to release GDNF [12]. However, several concerns have been raised, related to the reduced rate of cellular survival within the implanted graft, as well as the presence of immune rejection of the grafted cells by the host tissue.

When compared to the existing strategies, the use of biodegradable and biocompatible microspheres for the controlled brain release of GDNF could represent an attractive alternative for several reasons. First of all, microparticles are prepared with biodegradable polymers that do not require removal once the treatment is finished. Secondly, brain biocompatibility of particles prepared with poly (lactic-co-glycolic) acid (PLGA) polymers has already been well established [13-16] and therefore the appearance of host immune reaction against injected microparticles is very unlikely. Finally, the drug release profile of PLGA microspheres brings another important advantage. Therefore, GDNF dosage could be diminished, leading to a reduction of the possible side effects. However, protein encapsulation is not an easy task due to the labile nature of these macromolecules. Among the methods described, multiple emulsion solvent evaporation technique $(\mathrm{W} / \mathrm{O} / \mathrm{W})$ is generally accepted as the most suitable to encapsulate proteins and peptides [17]. Nevertheless, proteins may lose their biological activity during the manufacturing process. Since shear stress and vortexing are avoided, multiple emulsion prepared by Total Recirculation One-Machine System (TROMS) may be a feasible way of overcoming protein denaturation during microparticle preparation [18]. TROMS technology also produces very homogeneous batches on a semi-industrial scale, which is of great interest considering future scalingup and industrial issues.

Different formulations loaded with glycosylated rat recombinant GDNF were previously analyzed to optimize the neurotrophic factor microencapsulation by TROMS technology, the stability of the protein during the manufacturing process and the drug release profile [19]. In the present work we move one step forward by testing the in vivo efficacy and safety of GDNF-loaded microspheres in a rodent model of PD. We are particularly interested in evaluating their ability to restore the dopaminergic innervation 
in a model of partial dopaminergic fiber depletion that mimics the situation encountered in PD patients. Rotational testing, histological assessment as well as antibody response to glycosylated GDNF were performed to analyze the effects of GDNF-loaded microparticles after implantation in parkinsonian rats.

\section{Materials and Methods}

\subsection{Materials}

Rat recombinant glycosylated GDNF was expressed and purified as previously described [20]. Recombinant insect cell-derived rat GDNF was purchased from SIGMA (Steinheim, Germany). GDNF enzyme linked immunosorbant assay kit (ELISA) was purchased from Promega (Madison, USA). Poly (lactic-co-glycolic) acid (PLGA) with a lactic:glycolic ratio of 50:50 RG 503H (MW $34 \mathrm{kDa})$ was provided by BoehringerIngelheim (Ingelheim, Germany). Dichloromethane, acetone, dimethilsulphoside and glycerine were obtained from Panreac Quimica S.A (Barcelona, Spain). Poly (vinyl alcohol) $88 \%$ hydrolyzed (MW: 125,000) was obtained from Polyscences, Inc (Warington, USA). The rat pheocromocytome PC-12 cells were purchased from American Type Culture Collection (ATCC) (Rockville, MD, USA). Normal goat serum, normal rabbit serum, biotinylated rabbit anti goat IgG and the Vectastain ABC kit was provided by Vector Laboratories (Burlingame, CA, USA). Triton X-100, ExtrAvidin ${ }^{\circledR}$ Peroxidase, mouse anti TH monoclonal antibody, 6-hydroxydopamine hydrochloride, 3,3'-diaminobenzidine, D-amphetamine sulphate, and rhodamin B isothiocyanate were from Sigma-Aldrich (Barcelona, Spain). DPX was obtained from BDH Chemicals (Poole, UK). $\mathrm{H}_{2} \mathrm{O}_{2}$ and paraformaldehyde were purchased from Merck (Barcelona, Spain). Carboxymethylcellulose and mannitol were obtained from Cooper Pharmaceutique (Melun, France). Polysorbate 80 was provided by Prolabo (Paris, France). Biotinylated goat anti mouse IgG was obtained from Jackson ImmunoResearch (West Grove, PA, USA). Goat anti GDNF antibody was purchased from R\&D systems (Minneapolis, MN, USA), rabbit anti GFAP antibody was obtained from DAKO (Trappes, France), mouse anti CD11b antibody was purchased from AbD Serotec (Oxford, England) and rat anti dopamine transporter monoclonal antibody were obtained from Chemicon International (Temecula, CA, USA). Donkey anti rabbit and donkey anti mouse coupled to Alexa ${ }^{\circledR} 488$ were from Molecular Probes (Eugene, OR, USA). Horseradish-peroxidase-conjugated goat anti-rat IgG, horseradish-peroxidaseconjugated donkey anti rabbit IgG and horseradish-peroxidase-conjugated sheep anti mouse IgG were from Amersham GE Healthcare (Buckinghamshire, UK). ophenylenediamine dihydrochloride were obtained from SIGMA (Saint Louis, MO, USA). The rabbit anti GDNF polyclonal antibody and the mouse anti GDNF monoclonal IgG1 antibody were purchased from Santa Cruz Biotechnology (Santa Cruz, CA, USA).

\subsection{Preparation of GDNF-loaded microspheres}

GDNF-loaded microparticles were prepared by solvent extraction/evaporation method using TROMS as previously described [19]. Briefly, the organic solution composed of 2 $\mathrm{ml}$ of dichlorometane:acetone (3:1) containing $100 \mathrm{mg}$ of Resomer RG 503H was injected through a needle with an inner gauge diameter of $0.17 \mathrm{~mm}$ at a ratio of 30 $\mathrm{mL} / \mathrm{min}$ into the inner aqueous phase $(200 \mu \mathrm{l})$. The inner aqueous phase contained 135 $\mu \mathrm{g}$ of GDNF in $10 \mathrm{mM}$ phosphate, $50 \mathrm{mM}$ sodium chloride (PBS), $\mathrm{pH} 7.9,5 \mathrm{mg}$ of HSA and $5 \mu \mathrm{l}$ of PEG 400 . Next, the primary emulsion $\left(\mathrm{W}_{1} / \mathrm{O}\right)$ was recirculated through the system for 3 min under a turbulent regime at a flow rate of $30 \mathrm{~mL} / \mathrm{min}$. The first emulsion was then injected into $30 \mathrm{~mL}$ of the external aqueous phase $\left(\mathrm{W}_{2}\right)$ composed of 
$1.5 \%$ PVA. The turbulent injection through the needle with an inner gauge diameter of $0.50 \mathrm{~mm}$ resulted in the formation of a multiple emulsion $\left(\mathrm{W}_{1} / \mathrm{O} / \mathrm{W}_{2}\right)$, which was further homogenized by circulation through the system for $4 \mathrm{~min}$. The $\mathrm{W}_{1} / \mathrm{O} / \mathrm{W}_{2}$ emulsion was stirred at $1000 \mathrm{rpm}$ at room temperature for at least 3 hours to allow solvent evaporation and microspheres formation. Finally, particles were washed with ultrapure water and freeze-dried. For fluorescence-labelled microparticles, rhodamin B isothiocyanate $(0.5$ $\mathrm{mg} / \mathrm{mL}$ ) was added to inner aqueous phase and microspheres were prepared as described above.

\subsection{Characterization of microspheres}

\subsubsection{Particle size analysis}

The mean particle size and size distribution of the microspheres were examined by laser diffractometry using a Mastersizer-S® (Malvern Instruments, Malvern, UK). Microspheres were dispersed in ultrapure water and analyzed under continuous stirring. The average particle size was expressed as the volume mean diameter in micrometers. Samples were read in triplicate.

\subsubsection{Particle morphology}

Both the microsphere shape and surface structure were evaluated by SEM using Zeiss DSM 940A microscope with a digital imaging capture system (DISS of Point Electronic $\mathrm{GmbH})$.

\subsubsection{Drug content}

The amount of GDNF encapsulated in the microspheres was determined by dissolving 5 $\mathrm{mg}$ of freeze-dried loaded particles in $1 \mathrm{ml}$ of dimethyl sulfoxide (DMSO). Previously, it was verified that DMSO did not affect GDNF stability. The quantity of GDNF was measured in triplicates by ELISA using the GDNF Emax ${ }^{\circledR}$ ImmunoAssay System according to the manufacturer's instructions.

\subsubsection{In vitro release of GDNF from PLGA microspheres}

GDNF-loaded microspheres $(1 \mathrm{mg}, \mathrm{n}=3)$ were resuspended by vortexing in $0.5 \mathrm{ml}$ of PBS, pH 7.4 containing $0.1 \% \mathrm{BSA}$ and $0.02 \% \mathrm{w} / \mathrm{w}$ sodium azide. Incubation took place in rotating vials at $37{ }^{\circ} \mathrm{C}$. At defined times ranging from $30 \mathrm{~min}$ to 40 days, samples were centrifuged at $25000 \mathrm{~g}$, for $15 \mathrm{~min}$. Due to the instability of the protein in the release medium, the amount of drug released was indirectly determined by measuring the quantity of GDNF remaining within the microspheres by ELISA. Release profiles were expressed in terms of cumulative release, and plotted versus time.

\subsubsection{In vitro bioactivity assay}

The bioactivity of released GDNF was assessed using the PC-12 cell line as described previously [20]. These cells differentiate to a neural phenotype in response to neurotrophic factors such as GDNF or NGF [20-21]. PC-12 cells were plated onto 12 well culture plate at a low density, $2 \times 10^{3}$ cells $/ \mathrm{cm}^{2}$ in $1 \mathrm{ml}$ of culture media. The culture medium was supplemented 24 hours later with $50 \mathrm{ng}$ of GDNF released from microspheres over 24 hours, which had previously been quantified by ELISA. Neurite outgrowth was visualized after 7 days in culture under phase contrast illumination with a Leika DM IRB inverted microscope connected to a Hamamatsu ORCA-ER digital camera. PC-12 cells incubated with $50 \mathrm{ng} / \mathrm{ml}$ of purified rat recombinant GDNF were used as a positive control of the technique. PC-12 cells incubated with medium 
supplemented with the released medium from non-loaded microspheres were used as negative control of the technique.

\subsection{In vivo efficacy of GDNF brain delivery through PLGA microspheres}

A total number of 15 female Sprague-Dawley rats (Harlan, Barcelona, Spain), with a body weight between 220 to $240 \mathrm{~g}$ at the beginning of the experiment, were used in this study. Animals were kept in standard animal facilities with free access to food and water, in a temperature and humidity-controlled room with $12 \mathrm{~h}$ on-off light cycle. Animal handling was conducted at all times in compliance with the regulations of the Ethical Committee of the University of Navarra as well as with the European Community Council Directive Ref. 86/609/EEC.

\subsubsection{6-OHDA lesion surgery}

Animals were deeply anesthetized via an i.p. injection of a 1:1 mixture of ketamine (75 $\mathrm{mg} / \mathrm{kg}$ ) and xylazine $(10 \mathrm{mg} / \mathrm{kg}$ ). Rats were then placed in a stereotaxic frame (David Kopf, Tujunja, CA, USA). A total dose of $20 \mu \mathrm{g}$ of the neurotoxin 6-hydroxydopamine (6-OHDA) dissolved in $10 \mu \mathrm{l}$ of saline with $0.1 \%$ ascorbic acid was injected at two sites (equally spaced $1 \mathrm{~mm}$ apart; final concentration of $10 \mu \mathrm{g} / 5 \mu \mathrm{l}$ each) into the right striatum [22]. The injection rate was $1 \mu \mathrm{l} / \mathrm{min}$ and the needle was left in place for an additional $5 \mathrm{~min}$ before withdrawal. The stereotaxic coordinates used to perform the 6OHDA lesion were taken from the atlas of Paxinos and Watson [23]. Coordinates for the first injection were $0.5 \mathrm{~mm}$ rostral to bregma, $-2.5 \mathrm{~mm}$ lateral from the midline and $5 \mathrm{~mm}$ ventral from the dura surface, whereas the coordinates for the second injection were $-0.5 \mathrm{~mm}$ rostral to bregma, $-4.2 \mathrm{~mm}$ lateral from the midline and $-5 \mathrm{~mm}$ ventral from the dura surface.

\subsubsection{Microspheres implantation}

For stereotaxic implantation, freeze-dried microspheres were dispersed in a sterile aqueous medium consisting of $0.1 \%(\mathrm{w} / \mathrm{v})$ carboxymethylcellulose, $0.8 \%(\mathrm{w} / \mathrm{v})$ polysorbate 80 and $0.8 \%(\mathrm{w} / \mathrm{v})$ mannitol in PBS, $\mathrm{pH} 7.4$, for stereotaxic implantation. Surgery was performed 2 weeks after the 6-OHDA lesion under aseptic conditions using the same stereotaxic coordinates. All the injections were performed at a flow rate of $1 \mu \mathrm{L} / \mathrm{min}$. The syringe was left in place for 5 additional minutes to avoid the suspension to be expelled from the brain. Each rat received a total dose of $2.5 \mu \mathrm{g}$ of GDNF in two implantations, each comprising $1.5 \mathrm{mg}$ of microparticles in $8 \mu \mathrm{l}$ of dispersing medium. Control rats received the same amount of non-loaded microspheres and sham-operated animals received 2 injections of $8 \mu 1$ of dispersing medium.

\subsection{Behavioral test; drug induced rotation}

The animal rotational behavior induced after an i.p. injection of $5 \mathrm{mg} / \mathrm{ml} \mathrm{D}$ amphetamine was assessed on a computerized rotometer (Panlab, Barcelona, Spain) 13 days after 6-OHDA administration and 2, 4, 6, and 8 weeks post- treatments. Left and right full-body turns were monitored over a $90 \mathrm{~min}$ period. Net rotational asymmetry score was calculated by subtracting contralateral turns from ipsilateral turns and was expressed as full-body turns per minute in the direction ipsilateral to the lesion.

\subsection{Histology}

\subsubsection{Tissue processing}

At the end of the experiment animals were anesthetized with an overdose of $10 \%$ chloral hydrate in distilled water and then perfused transcardially with saline Ringer's 
solution followed by $500 \mathrm{ml}$ of cold fixative containing 4\% paraformaldehyde in 0.125 $\mathrm{M}$ phosphate buffer (PB) $\mathrm{pH}$ 7.4. The brains were removed and post-fixed for an additional period of $2 \mathrm{~h}$ in the same fixative solution and next transferred into a cryoprotective solution containing $20 \%$ glycerol and $2 \%$ dimethylsulphoxide in 0.125 M PB pH 7.4. Frozen coronal sections (30 $\mu \mathrm{m}$ thick) were obtained in a sliding microtome and collected in cryoprotective solution in 8 series of adjacent sections. Sections were stored at $-80{ }^{\circ} \mathrm{C}$ until further processing.

\subsubsection{Immunohistochemistry}

Immunohistochemistry was performed using antibodies against $\mathrm{TH}$ (mouse antiTH monoclonal antibody used at 1:2000), GDNF (goat anti GDNF antibody used at 1:100), Glial fibrillary acidic protein GFAP (rabbit antiGFAP polyclonal antibody used at $1: 1000$ ) and CD11b (mouse antiCD11b monoclonal antibody used at 1:500). For TH and GDNF immunohistochemistry, biotinylated goat anti mouse IgG (used at 1:1200) and biotinylated rabbit anti goat IgG (used at 1:1200) were used as secondary antibodies respectively. Sections were next incubated in a 1:5000 solution of peroxidaseconjugated ExtrAvidin $^{\circledR}$ and immunoreactive structures were visualized after incubation in $0.005 \% 3,3^{\prime}$-diaminobenzidine (DAB) and $0.001 \% \mathrm{H}_{2} \mathrm{O}_{2}$. Sections were dehydrated, cleared in xylene, and coverslipped with DPX. For GFAP and CD11b immunohistochemistry, sections were incubated with the secondary antibody donkey anti rabbit or donkey anti mouse coupled to Alexa ${ }^{\circledR} 488$ respectively (used at 1:250), rinsed mounted and dehydrated.

\subsection{Fiber density measurements}

The optical densities (OD) of the TH-immunoreactive fibers in the striatum were measured using a computerized image analysis system $\left(\right.$ AnalySIS $^{\circledR}$ ) 3.1, Soft Imaging Systems) reading optical densities as gray levels. For each animal, the optical density was measured at three different rostrocaudal levels along the striatum according to Paxinos and Watson: (1) rostral striatum (bregma $+1 \mathrm{~mm}$ ); (2) medial striatum (bregma $-0.5 \mathrm{~mm}$ ); and (3) caudal striatum (bregma $-1 \mathrm{~mm}$ ). A rectangle of $410.000 \mu \mathrm{m}^{2}$ was placed on the most lateral region of the striatum and the OD was measured using an image analysis software (AnalySIS $\left.{ }^{\circledR}\right) 3.1$, Soft Imaging Systems). Optical density values for the striatum on the ipsilateral side were expressed as a percentage of the contralateral non-lesioned side.

\subsection{Evaluation of serum IgG antibody response against rat glycosylated GDNF}

Blood samples were obtained from the animal at the time of perfusion by heart puncture. To separate serum, blood was allowed to clot for 1 hour at $37^{\circ} \mathrm{C}$ and centrifuged at $3000 \mathrm{x} \mathrm{g}$ for $15 \mathrm{~min}$ at $20^{\circ} \mathrm{C}$. Serum aliquots were stored at $-80^{\circ} \mathrm{C}$. For the detection of anti-rat GDNF antibodies, 96-well microtiter plates were coated with $50 \mathrm{ng} /$ well of recombinant rat GDNF in $50 \mu \mathrm{L}$ carbonate buffer $(0,025 \mathrm{M}$ sodium bicarbonate, $0,025 \mathrm{M}$ sodium carbonate) $\mathrm{pH} 8.2$, per well overnight at $4{ }^{\circ} \mathrm{C}$. After washing with PBS the wells were blocked with blocking buffer (1\% BSA, 0,1\% Tween$20, \mathrm{pH} 7.4$ in PBS) for 1 hour at room temperature. Plates were incubated with 50 $\mu \mathrm{L} /$ well of serum samples diluted $1: 10^{2}$ and $1: 10^{4}$ in blocking buffer for 2 hours at room temperature. After washing, plates were incubated for 30 minutes at room temperature with $50 \mu \mathrm{L} /$ well of $1: 10^{3}$ horseradish-peroxidase-conjugated goat anti-rat $\operatorname{IgG}$ in blocking buffer, washed, and visualized by incubating with $100 \mu \mathrm{L}$ solution ophenylenediamine dihydrochloride. Absorbance at $450 \mathrm{~nm}$ was measured after 30 
minutes. For each sample duplicate determinations of each sample were performed. Background correction was determined for each sample using uncoated wells.

\subsection{Statistical analysis}

All values were expressed as the mean \pm s.e. (standard error). Comparisons between groups were carried out using one-way ANOVA with Tukey post hoc analysis with and SPSS vs 13.0 software.

\section{Results}

\subsection{Microspheres characterization}

GDNF-loaded microparticles were successfully prepared by $\mathrm{W}_{1} / \mathrm{O} / \mathrm{W}_{2}$ emulsion/extraction process using TROMS technology. The mean particle size measured by laser diffractometry was $8.42 \pm 0.07 \mu \mathrm{m}$, which was totally compatible with a stereotaxic injection according with previous studies [13]. The total amount of loaded GDNF was $68 \mu \mathrm{g}$ per $100 \mathrm{mg}$ of polymer, suitable for in vivo studies. The yield of the fabrication process was $82 \%$. SEM analysis showed that GDNF-PLGA microspheres were spherical in shape and with a smooth surface (Figure 1A).

\subsection{In vitro release kinetics}

In vitro release kinetics was performed in PBS (pH 7.4) at $37^{\circ} \mathrm{C}$ for 40 days (Figure 1B). After an initial burst, a continuous GDNF release was observed from day 1 to 14, in which drug diffuses through the polymer. Finally, a second increase in the rate of release was observed from day 14 to 40 probably caused by polymer degradation. Indeed, $67 \%$ of the total GDNF was released within the first 40 days.

\subsection{In vitro bioactivity assay}

PC-12 cells derive from a rat pheochromocytome and present an undifferentiated aspect when grown in culture. When bioactive GDNF is added to the culture medium, the cells differentiate and start to develop a neural phenotype visualized by the presence of neuronal-like processes. As shown in Figure 1C, no outgrowth of neurites was observed in the cells treated with the release medium from non-loaded microspheres. By contrast, $50 \mathrm{ng}$ of GDNF released from microspheres were able to differentiate PC-12 cells after 7 days of treatment showing that the released neurotrophic factor remains biologically active after the microencapsulation process (Figure 1D). A similar differentiation was observed in cells treated with the same amount of purified rat recombinant GDNF (Figure 1E). These results demonstrated that the encapsulated GDNF was biologically bioactive.

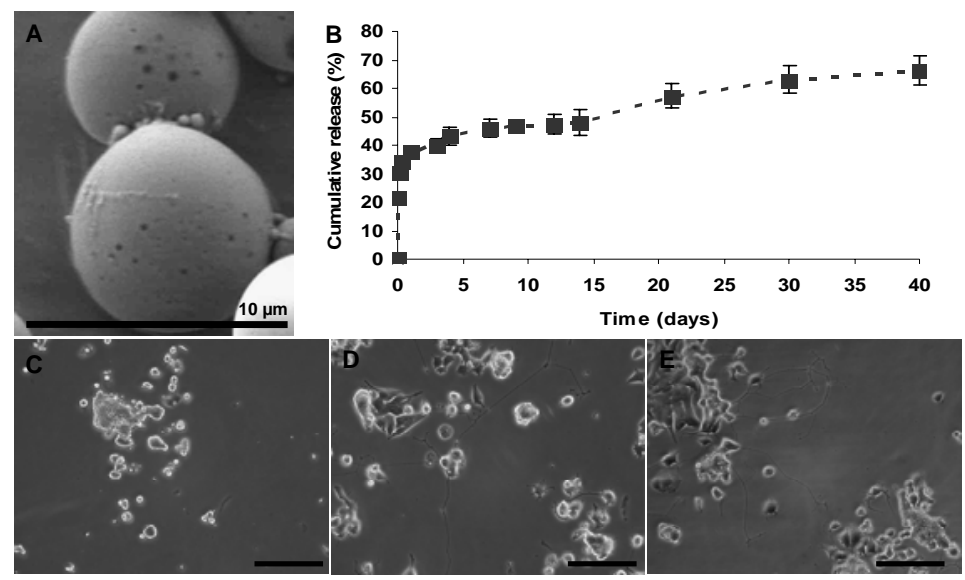


Figure 1: (A) Scanning electron micrographs of representative GDNF-loaded PLGA microparticles prepared by TROMS technology. (B) GDNF in vitro release profile from PLGA microspheres $(\mathrm{n}=3)$. (C-E) Assessment of the biological activity of GDNF released from microparticles. Phase-contrast microscopy of cells that were cultured for 7 days in medium supplemented (C) with the release medium of non-loaded microspheres, (D) with GDNF released over 24 hours from loaded microparticles and (E) with the same amount of purified rat recombinant GDNF. Note the appearance of neurites both in D also in E demonstrating the bioactivity of GDNF released from microspheres. Bar length $100 \mu \mathrm{m}$

\subsection{In vivo experiments}

\subsubsection{Functional effects of GDNF-loaded microparticles implanted into the striatum}

The ultimate proof-of-concept on the potential neurorestorative effect exerted by microencapsulated GDNF was evaluated in an animal model of partial dopaminergic depletion. The experimental design is summarized in Figure 2.

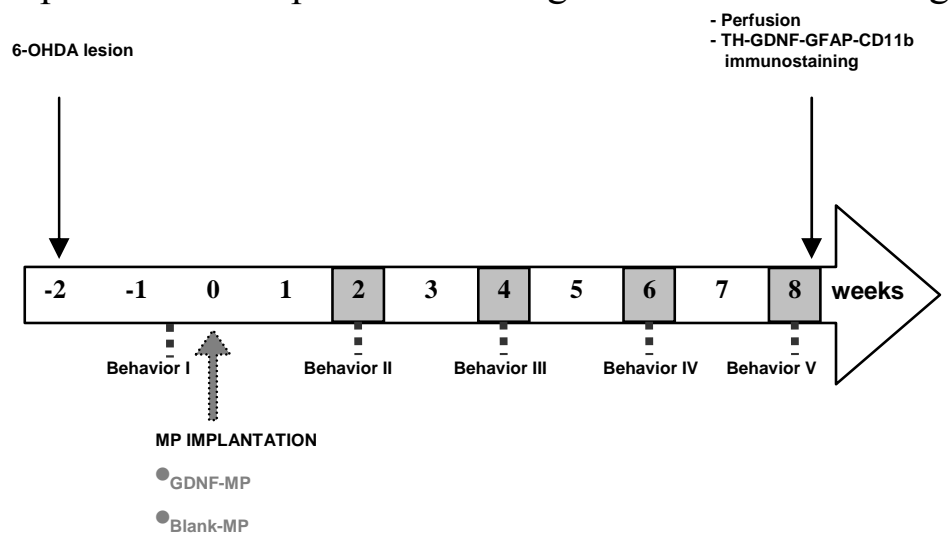

Figure 2: Time course of the experiments. Animals received GDNF-loaded microparticles, non-loaded microparticles or microspheres dispersing medium 2 weeks after 6-OHDA lesion. Rotational behavior was analyzed before microspheres implantation and at 2, 4, 6 and 8 weeks post-implantation. At this time, animals were sacrificed to perform immunohistochemical analysis.

The intraparenchymal delivery of the neurotoxin 6-OHDA resulted in a successful partial dopaminergic denervation within the striatum, appropriate to evaluate the efficacy of GDNF-loaded microspheres. Amphetamine-induced motor behavior was taken as an indicator of the degree of dopaminergic lesion resulting from the 6-OHDA delivery. Only rats showing more than 6 turns ipsilateral to the lesion side per minute were considered as properly lesioned and then used for microspheres implantation. All rats survived the implantation of microspheres, which was well tolerated by the animals. Rats were tested with amphetamine 2, 4, 6 and 8 weeks after microparticles implantation. The evolution in time of the mean score of amphetamine-induced rotations of the animals is shown in Figure 3. No statistically significant differences in the number of rotations between groups were observed before microparticle implantation. Our data showed that, during the 2 months analyzed, the administration of either sham or non-loaded microspheres did not cause any significant change in the number of turns ipsilateral to the lesion per minute (Sham from $11.48 \pm 1.5$ to $11.49 \pm$ 1.70 and non-loaded microspheres from $10.02 \pm 1.4$ to $10.75 \pm 2.9$ ). By contrast, animals treated with GDNF-loaded microspheres showed a gradual reduction in the number of ipsilateral turns per minute, from $9.5 \pm 0.7$ before microparticle implantation to $1.5 \pm 1$ two months post-treatment. The decrease has been noticed 4 weeks after microsphere implantation. At this point, the number of turns per minute induced by amphetamine was reduced by 28\%. Marked differences were more evident 6 weeks after treatment, and at this point the scores reached statistical significance when compared both to sham and to non loaded-microsphere treated animals $(\mathrm{p}<0.05)$. Two months after treatment, the reduction in the number of ipsilateral turns was still 
maintained achieving a total abolition of amphetamine-induced rotation in GDNFtreated animals. Interestingly, it was observed that this score was even normalized (0.6, 0.1 and 0.015 turn per minute) for 3 rats in this group. At this time, the score of GDNF microsphere-treated animals was statistically different when compare both to sham and to non loaded-microsphere treated animals $(\mathrm{p}<0.05)$ (Figure 3).

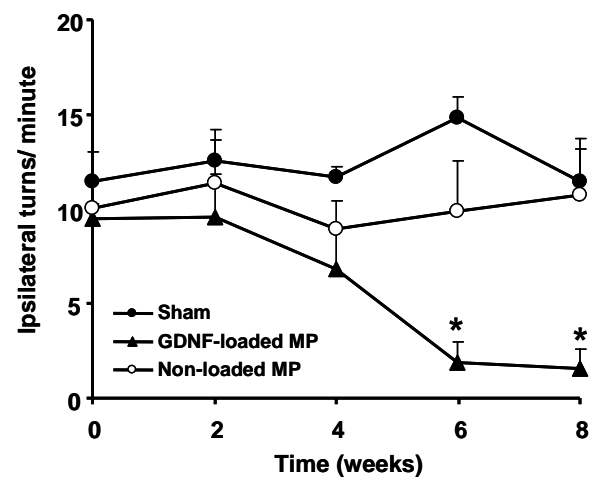

Figure 3: Changes in the amphetamine-induced rotational behavior after treatment with GDNF-loaded microspheres. There is a marked decrease on the number of net turns 4 weeks post-treatment. Rotational behavior markedly decreased 6 weeks after GDNF treatment and this recovery was still maintained 8 weeks post-treatment. The administration of either the microspheres dispersing medium (sham) or empty microspheres has no impact on the rotational behavior observed after i.p. injection of $5 \mathrm{mg} / \mathrm{Kg}$ of amphetamine. Each bar represents the mean value \pm S.E. $(* p<0.05$ compared to the non-loaded microparticles treated group and to the animals that received the microparticles dispersing medium)

\subsubsection{Histological effects of striatal GDNF microparticle implantation}

Animals were sacrificed 8 weeks after treatment to verify whether the improvement in the behavioral test was accomplished by GDNF induced reinnervation of dopaminergic fibers in the striatum. Brain sections were stained immunocytochemically for $\mathrm{TH}$, a marker for dopaminergic neurons (Figure 4). The striatum of animals treated with GDNF-loaded microparticles showed a clear increase in TH-immunostaining (Figure 4A"), which was especially prominent in the area close to the injection site of the particles. The striatal reinnervation was due to the GDNF delivery since this effect was not observed in the animal groups treated with the empty microspheres (Figure 4B") or with the dispersing medium (Figure 4C").

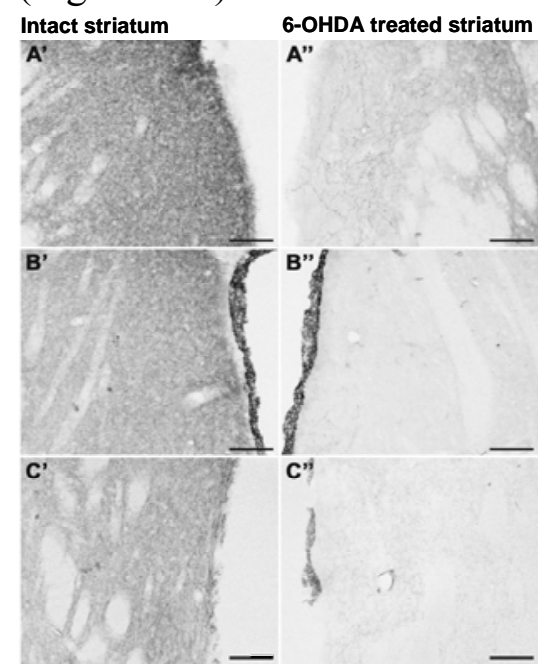


Figure 4: Photomicrographs of striatums immunostained for TH from intact hemispheres (A', $B^{\prime}$ and $\left.C^{\prime}\right)$ and 6-OHDA lesioned hemispheres injected with GDNF-loaded microspheres (A"), non-loaded microspheres (B") and dispersium medium ( $\left.\mathrm{C}^{\prime \prime}\right) 15$ days after the partial dopaminergic lesion. The striatal reinnervation induced by the 8-week treatment with microencapsulated GDNF was characterized by the presence of dense plexus of TH immunoreactive fibers (A"). Such a substantial striatal reinnervation has never been noticed in the animal groups treated with empty microspheres (B') or with the dispersing medium (C"). Scale bar is $100 \mu \mathrm{m}$.

The increase in TH-immunostaining in animals treated GDNF-loaded microspheres was quantified by measuring the OD at rostral, medial and caudal levels of the striatum. OD values for the striatum on the ipsilateral side were expressed as a percentage of the contralateral non-lesioned side. The density of TH-immunopositive fibers in the striatum of sham and non-loaded microspheres was approximately $60 \%$ of that in the non-lesioned striatum in the three levels analyzed (Figure 5). In the GDNF treated animals, the OD was $77.44 \pm 8.24 \%, 91.75 \pm 9.69 \%$ and $95 \pm 5.74 \%$ in the rostral, medial and caudal sections respectively, which were significantly different $(p<0.05)$ when compared to animals treated with non-loaded microspheres and with sham in the three levels analyzed. Moreover, when comparing sections of GDNF treated animals, quantitative densitometry revealed that the most abundant reinnervation was noticed within medial and caudal levels of the striatum, which corresponded to the levels located closer to the microparticle injection sites (Figure 5). This probably reflects the important diffusion of GDNF from microparticles at these levels.

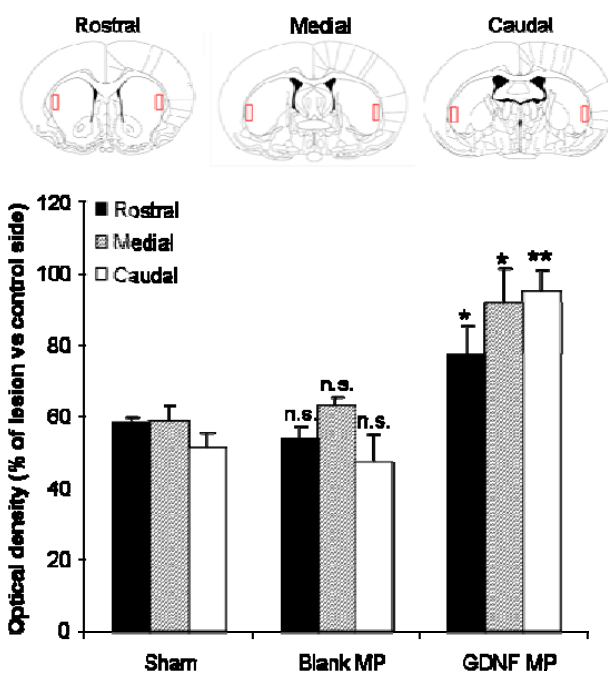

Figure 5: Changes in the optical density (OD) at the three levels of the striatum analyzed, expressed as the percentage of lesion versus the control side, as result of the 8-week treatment with microencapsulated GDNF. Significant increases in optical density of the TH stain were found in the striatum of animals treated with GDNF-loaded microspheres with respect to the other two groups $(* p<0.05)$. This effect was observed in the three levels of the striatum analyzed, and was more pronounced in medial and caudal striatal levels, the ones more closely located to the injection site. This is probably due to the diffusion of GDNF from microparticles at this level. Mean values \pm S.E.

Interestingly, non-loaded and GDNF-loaded microparticles were still visible by optical microscopy 2 months post-implantation. No evidence of damage was observed on the tissue that surrounds the implanted microparticles indicating that they were well tolerated. Consistent with previous reports, GFAP-positive reactive astrocytes were observed around the needle tract (Figure 6A) [14, 16, 24]. This reaction was the same for the 3 groups of animals demonstrating that the astrocytic response is attributed to the mechanical trauma that occurs during surgery and not to the polymer. No CD11b 
positive macrophages were found (Figure 6D). It was noticed that both GDNF-loaded microparticles and non-loaded microparticles were not totally degraded at this time.
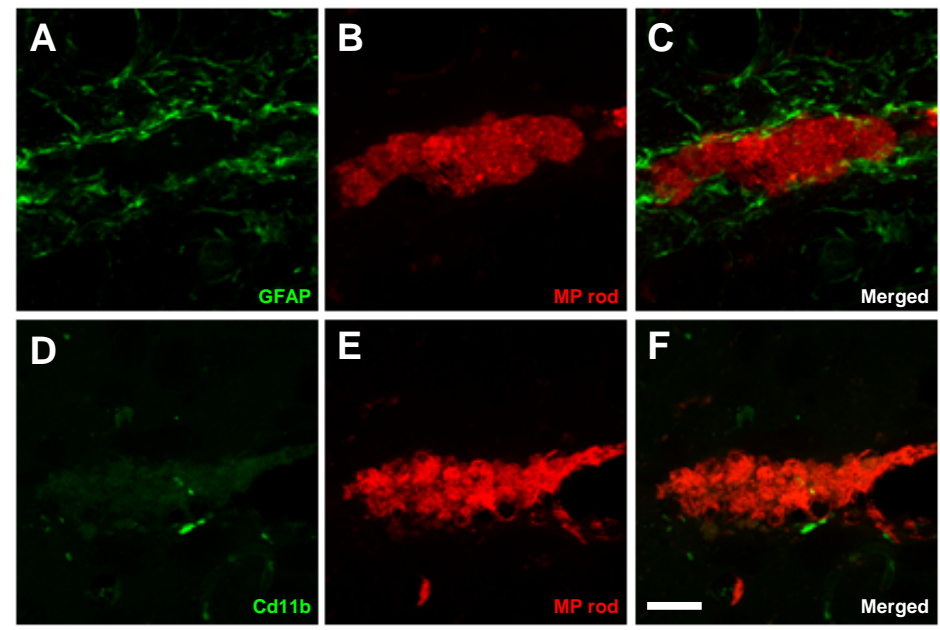

Figure 6: Confocal microscopy images of sections through the striatum 2 months after microparticles injection immunostained for GAFP (A) and CD11b (D) in green and adjacent striatal sections showing fluorescent rhodamine microparticles in red (B and $\mathrm{E})$. Histological analysis revealed a limited moderate astrocytic reaction in A where some astrocytes were stained with GFAP antibody. As can be observed in $\mathrm{C}$, which is the merge of $\mathrm{A}$ and $\mathrm{B}$, the astrocytic reaction was localized surrounding the microparticles injection site. This reaction was the same for the 3 groups of animals. No CD11b positive macrophages were found 2 months after microparticles implantation (D). We can note that microparticles were not totally degraded 2 months after implantation and that they were uniformly distributed along the injection site (B and E). Scale bar represents $20 \mu \mathrm{m}$.

Immunohistochemical detection of GDNF 5 weeks post-implantation showed immunoreactivity within the GDNF-microparticles injection site (Figure 7 A-A'). As shown in Figure 7, GDNF could still be detected 5 weeks following microparticles implantation, indicating that microparticles were suitable for long-term GDNF delivery.

\subsubsection{Evaluation of serum IgG antibody response against rat glycosylated GDNF}

To assess the systemic safety of rat glycosylated recombinant GDNF administration, serum GDNF antibodies levels were measured by ELISA in all the animals. Sera from GDNF-MP treated animals were negative for GDNF antibodies. The serum samples obtained from non-loaded treated animals and from sham-operated animals were also negative for GDNF antibodies.

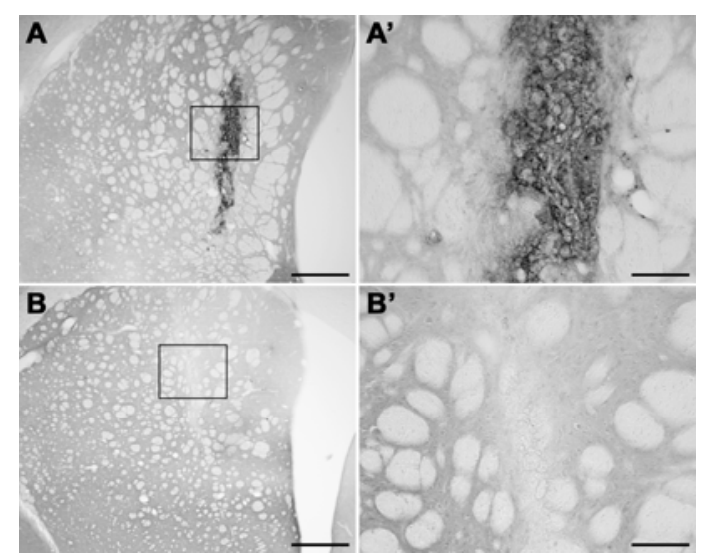

Figure 7: Low power photomicrographs $(\mathrm{A}, \mathrm{B})$ and insets $\left(\mathrm{A}^{\prime}, \mathrm{B}^{\prime}\right)$ of striatal sections immunostained for GDNF of rats treated with GDNF-loaded microspheres (A-A') and rats treated with non-loaded 
microspheres $\left(\mathrm{B}, \mathrm{B}^{\prime}\right)$ at 5 weeks after treatment. The immunohistochemistry for GDNF showed the deposit of GDNF-loaded microspheres within the striatum (A, $\left.\mathrm{A}^{\prime}\right)$ which is more evident in the inset $\mathrm{A}^{\prime}$. GDNF-immunoreactive microparticles were still visible after five weeks post-injection. Scale bar is 500 $\mu \mathrm{m}$ in panel $\mathrm{A}$ and $\mathrm{B}$, and $100 \mu \mathrm{m}$ in panel $\mathrm{A}^{\prime}$ and $\mathrm{B}^{\prime}$.

\section{Discussion}

The present paper is a proof-of-concept study, designed to assess the potential neurorestorative properties of microencapsulated GDNF within biodegradable and biocompatible PLGA microspheres. The trophic effect of GDNF on the dopaminergic system is widely acknowledged in the current bibliography [1, 2]. However, the potential value of this protein for PD treatment is hindered by several safety and efficacy concerns related to the brain delivery strategies attempted so far. In this regard, there is an urgent need to develop a safe and effective GDNF brain delivery system, as was emphasized in a recent paper where the use of GDNF for PD treatment was discussed [25]. An ideal vector or device for GDNF brain delivery must meet several criteria, such as, compatibility with the host tissue, no immunological reaction, minimal damage at the injection site and controlled release of the protein to the CNS. Furthermore, in an attempt to improve the biological activity and to reduce adverse effects such as the generation of blocking antibodies against GDNF, the administered neurotrophic factor should closely mimic the endogenous protein glycosylation pattern. Although none of the existing strategies for GDNF brain delivery fulfill these demanding criteria, microencapsulated GDNF within PLGA microspheres comes closer to meeting these requirements.

Jollivet et al. proposed for the first time the brain delivery of GDNF using PLGA microspheres in order to induce dopaminergic reinnervation in a partial model of PD [26]. Parkinsonian rats treated with microparticles loaded with the nonglycosylated protein, experimented functional improvements accompanied with neural regeneration. Consequently, it was demonstrated that microparticles were appropriate to deliver the neurotrophic factor into the CNS [26, 27]. In this present work, notable modifications have been made in order to enhance the effect of microencapsulated GDNF and to reduce some safety concerns.

First, and most significant, was the use of highly purified GDNF with a glycosylation pattern similar to the endogenous protein [20]. Thus, injection of impurities to the CNS was avoided and production of antibodies against the neurotrophic factor was prevented. This aspect is crucial because one of the safety concerns which halted the phase II clinical trials in which patients received a protein expressed in E. coli, was the detection of antibodies against GDNF in $10 \%$ of the patients when the protein was injected through an infusion pump [6]. Antibodies could be easily generated against exogenous GDNF produced in bacteria, which differs from the endogenous protein in its glycosylation pattern [28]. No antibody response to rat GDNF was detected in this work, where glycosylated recombinant GDNF had been used.

The second modification made was improving the preparation method of the microparticles. In this present study, particles were prepared by TROMS technology. This system that involves the injection of the phases under a turbulent regime is ideal for encapsulation of complex and fragile bioactive molecules. Shear forces such as ultrasound or high pressure homogenizers are avoided allowing for proteins to remain biologically active during the manufacturing process [18]. In the present study, GDNF bioactivity was preserved throughout the process as demonstrated by the in vitro differentiation of PC-12 cells. These results confirmed TROMS technology as an appropriate method for the microencapsulation of fragile therapeutic agents such as neurotrophic factors. Furthermore, another significant benefit of this innovative system 
in view of its industrial application is the consistent production of very homogeneous batches of microparticles allowing for an easy scale-up of the manufacturing process.

Another improvement is the reduction of the microparticle injection volume required for implantation into the brain. Our study used 20\% less injection volume compared to others who used larger volumes for an even distribution of microparticles within the tissue [24, 26, 27]. Despite the small volume of injection used, microspheres were uniformly distributed along the site of injection (Figure 6 and 7). The volume of injection is a crucial safety factor as reduction in injection volume may reduce the mechanical trauma, non-specific lesions and animal behavior deficits due to microspheres administration. Interestingly, in this work and in contrast to previous studies [26], results from amphetamine-induced rotational behavior indicate that behavior of experimental animals did not deteriorate over time (Figure 3).

As a result of these variations, microparticles prepared by TROMS and loaded with glycosylated GDNF exhibited excellent in vivo efficacy and safety with a consistent improvement in behavior, significantly different from both controls and non-loaded microparticles 6 weeks after implantation. Furthermore, $60 \%$ of the animals treated with GDNF-loaded microparticles fully recovered from their rotational asymmetry 8 weeks after treatment and $20 \%$ exhibited less than 2 turns per minute at the same time. The motor behavior restoration was accompanied with a higher fiber density in the GDNF treated striatum, as Jollivet et al previously described [26], making this strategy effective for delivering GDNF in the striatum of hemiparkinsonian rats. The data presented in this article offers valuable evidence that the enhanced in vivo neurorestorative effect observed is likely to be the result of the improvements discussed.

\section{Acknowledgements}

We thank E. Roda, C. Molina and L. Sindji for technical support. This project was funded by the Department of Health and Education of the Government of Navarra, by the MAPFRE Medicine Foundation, by the CAN Foundation and by the UTEproject/Foundation for Applied Medical Research (F.I.M.A.). E. Garbayo is the beneficiary of a pre-doctoral fellowship from the Government of Navarra.

\section{References}

1. L.F. Lin, D.H. Doherty, J.D. Lile, S. Bektesh, F. Collins, GDNF: a glial cell linederived neurotrophic factor for midbrain dopaminergic neurons. Science 260(5111) (1993) 1130-1132.

2. R. Grondin, D.M. Gash, Glial cell line-derived neurotrophic factor (GDNF): a drug candidate for the treatment of Parkinson's disease. J Neurol 245(11 Suppl 3) (1998) P35-42.

3. S.S. Gill, N.K. Patel, G.R. Hotton, K. O'Sullivan, R. McCarter, M. Bunnage, D.J. Brooks, C.N. Svendsen, P. Heywood, Direct brain infusion of glial cell line-derived neurotrophic factor in Parkinson disease. Nat Med 9(5) (2003) 589-595.

4. N.K. Patel, M. Bunnage, P. Plaha, C.N. Svendsen, P. Heywood, S.S. Gill, Intraputamenal infusion of glial cell line-derived neurotrophic factor in PD: a two-year outcome study. Ann Neurol 57(2) (2005) 298-302.

5. J.T. Slevin, G.A. Gerhardt, C.D. Smith, D.M. Gash, R. Kryscio, B. Young, Improvement of bilateral motor functions in patients with Parkinson disease through the unilateral intraputaminal infusion of glial cell line-derived neurotrophic factor. $\mathrm{J}$ Neurosurg 102(2) (2005) 216-222.

6. A.E. Lang, S. Gill, N.K. Patel, A. Lozano, J.G. Nutt, R. Penn, D.J. Brooks, G. Hotton, E. Moro, P. Heywood, M.A. Brodsky, K. Burchiel, P. Kelly, A. Dalvi, B. Scott, 
M. Stacy, D. Turner, V.G. Wooten, W.J. Elias, E.R. Laws, V. Dhawan, A.J. Stoessl, J. Matcham, R.J. Coffey, M. Traub, Randomized controlled trial of intraputamenal glial cell line-derived neurotrophic factor infusion in Parkinson disease. Ann Neurol 59(3) (2006) 459-466.

7. M. Hutchinson, S. Gurney, R. Newson, GDNF in Parkinson disease: an object lesson in the tyranny of type II. J Neurosci Methods 163(2) (2007) 190-192.

8. S. Behrstock, A. Ebert, J. McHugh, S. Vosberg, J. Moore, B. Schneider, E. Capowski, D. Hei, J. Kordower, P. Aebischer, C.N. Svendsen, Human neural progenitors deliver glial cell line-derived neurotrophic factor to parkinsonian rodents and aged primates. Gene Ther 13(5) (2006) 379-388.

9. J.H. Kordower, M.E. Emborg, J. Bloch, S.Y. Ma, Y. Chu, L. Leventhal, J. McBride, E.Y. Chen, S. Palfi, B.Z. Roitberg, W.D. Brown, J.E. Holden, R. Pyzalski, M.D. Taylor, P. Carvey, Z. Ling, D. Trono, P. Hantraye, N. Deglon, P. Aebischer, Neurodegeneration prevented by lentiviral vector delivery of GDNF in primate models of Parkinson's disease. Science 290(5492) (2000) 767-773.

10. S. Palfi, L. Leventhal, Y. Chu, S.Y. Ma, M. Emborg, R. Bakay, N. Deglon, P. Hantraye, P. Aebischer, J.H. Kordower, Lentivirally delivered glial cell line-derived neurotrophic factor increases the number of striatal dopaminergic neurons in primate models of nigrostriatal degeneration. J Neurosci 22(12) (2002) 4942-4954.

11. A. Eslamboli, B. Georgievska, R.M. Ridley, H.F. Baker, N. Muzyczka, C. Burger, R.J. Mandel, L. Annett, D. Kirik, Continuous low-level glial cell line-derived neurotrophic factor delivery using recombinant adeno-associated viral vectors provides neuroprotection and induces behavioral recovery in a primate model of Parkinson's disease. J Neurosci 25(4) (2005) 769-777.

12. L. Grandoso, S. Ponce, I. Manuel, A. Arrue, J.A. Ruiz-Ortega, I. Ulibarri, G. Orive, R.M. Hernandez, A. Rodriguez, R. Rodriguez-Puertas, M. Zumarraga, G. Linazasoro, J.L. Pedraz, L. Ugedo, Long-term survival of encapsulated GDNF secreting cells implanted within the striatum of parkinsonized rats. Int J Pharm 343(1-2) (2007) 69-78. 13. M.J. Blanco-Prieto, C. Durieux, V. Dauge, E. Fattal, P. Couvreur, B.P. Roques, Slow delivery of the selective cholecystokinin agonist pBC 264 into the rat nucleus accumbens using microspheres. J Neurochem 67(6) (1996) 2417-2424.

14. D.F. Emerich, M.A. Tracy, K.L. Ward, M. Figueiredo, R. Qian, C. Henschel, R.T. Bartus, Biocompatibility of poly (DL-lactide-co-glycolide) microspheres implanted into the brain. Cell Transplant 8(1) (1999) 47-58.

15. J. Veziers, M. Lesourd, C. Jollivet, C. Montero-Menei, J.P. Benoit, P. Menei, Analysis of brain biocompatibility of drug-releasing biodegradable microspheres by scanning and transmission electron microscopy. J Neurosurg 95(3) (2001) 489-494.

16. E. Fournier, C. Passirani, C.N. Montero-Menei, J.P. Benoit, Biocompatibility of implantable synthetic polymeric drug carriers: focus on brain biocompatibility. Biomaterials 24(19) (2003) 3311-3331.

17. U. Bilati, E. Allemann, E. Doelker, Strategic approaches for overcoming peptide and protein instability within biodegradable nano- and microparticles. Eur J Pharm Biopharm 59(3) (2005) 375-388.

18. G. del Barrio, F.J. Novo, J.M. Irache, Loading of plasmid DNA into PLGA microparticles using TROMS (Total Recirculation One-Machine System): evaluation of its integrity and controlled release properties. J Control Release 86(1) (2003) 123-130.

19. E. Garbayo, E. Ansorena, J.L. Lanciego, M.S. Aymerich, M.J. Blanco-Prieto, Sustained release of bioactive glical cell-line derived neurotrophic factor from biodegradable polymeric microspheres. Eur J Pham Biophar 69 (3) (2008) 844-851. 
20. E. Garbayo, E. Ansorena, J.L. Lanciego, M.S. Aymerich, M.J. Blanco-Prieto, Purification of bioactive glycosylated recombinant glial cell line-derived neurotrophic factor. Int J Pharm 344 (1-2) (2007) 9-15.

21. X. Cao, M.S. Schoichet, Delivering neuroactive molecules from biodegradable microspheres for application in central nervous system disorders. Biomaterials 20(4) (1999) 329-339.

22. D. Kirik, C. Rosenblad, A. Bjorklund, Characterization of behavioral and neurodegenerative changes following partial lesions of the nigrostriatal dopamine system induced by intrastriatal 6-hydroxydopamine in the rat. Exp Neurol 152(2) (1998) 259-277.

23. G. Paxinos, C. Watson, The Rat Brain in Stereotaxic Coordinates. Academic Press. Orlando (1996).

24. P. Menei, J.M. Pean, V. Nerriere-Daguin, C. Jollivet, P. Brachet, J.P. Benoit, Intracerebral implantation of NGF-releasing biodegradable microspheres protects striatum against excitotoxic damage. Exp Neurol 161(1) (2000) 259-272.

25. M. Hong, K. Mukhida and I. Mendez, GDNF therapy for Parkinson's Disease. Expert Rev Neurother 8(7) (2008) 1125-1139.

26. C. Jollivet, A. Aubert-Pouessel, A. Clavreul, M.C. Venier-Julienne, S. Remy, C.N. Montero-Menei, J.P. Benoit, P. Menei, Striatal implantation of GDNF releasing biodegradable microspheres promotes recovery of motor function in a partial model of Parkinson's disease. Biomaterials 25(5) (2004) 933-942.

27. C. Jollivet, A. Aubert-Pouessel, A. Clavreul, M.C. Venier-Julienne, C.N. MonteroMenei, J.P. Benoit, P. Menei, Long-term effect of intra-striatal glial cell line-derived neurotrophic factor-releasing microspheres in a partial rat model of Parkinson's disease. Neurosci Lett 356(3) (2004) 207-210.

28. T.B. Sherer, B.K. Fiske, C.N. Svendsen, A.E. Lang, J.W. Langston, Crossroads in GDNF therapy for Parkinson's disease. Mov Disord 21(2) (2006) 136-141. 